INPLASY

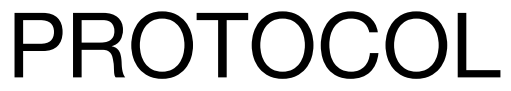

To cite: Pan et al. Acupuncture and combination therapy in the treatment of postpartum depression: a meta-analysis. Inplasy protocol 2021100047. doi:

10.37766/inplasy2021.10.0047

Received: 15 October 2021

Published: 15 October 2021

Corresponding author:

Youbing Xia

xybd1968@sina.com

Author Affiliation:

Nanjing University of Chinese

Medicine

\section{Support: NSFC81873371,} KYCX21-1693.

Review Stage at time of this submission: Data analysis.

Conflicts of interest:

None declared.

\section{Acupuncture and combination therapy in the treatment of postpartum depression: a meta-analysis}

Pan, Y1; Xi, J2; Liu, J3; Shen J4; Cheng, J5; Xia, Y6.

Review question / Objective: P: Patients with postpartum depression. I: The treatment group will be dominated by acupuncture (including electroacupuncture, warm acupuncture, ear acupuncture, etc.), acupuncture combined with drug therapy or psychotherapy, acupoint selection, acupuncture manipulation, needle retention time and course of treatment are not limited. C: The control group will be a single use of medium and Western medicine, psychotherapy or comfort therapy. O: The primary outcome indicator will be the effective rate(ER), and the secondary outcome indicators will be the Hamilton depression scale (HAMD) score, the Edinburgh postnatal depression scale(EPDS) score, and TCM syndromes Score, serum estradiol(E2) level, and occurrence of adverse reactions. The included study contains at least one outcome indicator.

Information sources: From databases of CNKI, WANGFANG, SinoMed, VIP, PubMed, Web of Science, MEDLINE, EBSCOhost, the Cochrane Library and OVID.

INPLASY registration number: This protocol was registered with the International Platform of Registered Systematic Review and Meta-Analysis Protocols (INPLASY) on 15 October 2021 and was last updated on 15 October 2021 (registration number INPLASY2021100047).

\section{INTRODUCTION}

Review question / Objective: P: Patients with postpartum depression. I: The treatment group will be dominated by acupuncture (including electroacupuncture, warm acupuncture, ear acupuncture, etc.), acupuncture combined with drug therapy or psychotherapy, acupoint selection, acupuncture manipulation, needle retention time and course of treatment are not limited. C: The control group will be a single use of medium and Western medicine, psychotherapy or comfort 
therapy. O: The primary outcome indicator will be the effective rate(ER), and the secondary outcome indicators will be the Hamilton depression scale (HAMD) score, the Edinburgh postnatal depression scale(EPDS) score, and TCM syndromes Score, serum estradiol(E2) level, and occurrence of adverse reactions. The included study contains at least one outcome indicator.

Condition being studied: Postpartum depression is a postpartum mental disorder. The main symptoms are depression and mental depression after delivery. In severe cases, hallucinations or suicide occur. The onset time is usually defined as 1 day to 12 months after delivery. As an important global health issue, PPD not only affects the physical and mental health of mothers, but also has a negative impact on family life and infant emotional cognition. According to reports, the prevalence of PPD in our country is $10 \%-15 \%$, with an average of $14.7 \%$. The occurrence of PPD is closely related to the rapid changes in sex hormone levels during the perinatal period. The current methods used to treat PPD include drug therapy, psychotherapy and physical therapy. Antidepressants can bring unknown risks to breastfed babies and have a potential negative impact on the development of children. Therefore, antidepressants are difficult to become the first choice for many women. Evidence-based medicine has shown that the effect of comprehensive treatment is better than any single treatment. The current expert consensus on the prevention and treatment of post-depressive disorder in our country recommends the principle of comprehensive treatment. Acupuncture therapy has been proven to be as effective as antidepressants and safer, and can be used as a complementary alternative therapy for the clinical treatment of PPD. Therefore, this study analyzes the clinical effectiveness and safety of acupuncture and acupuncture combination therapy in the treatment of PPD, and provides evidence-based medicine for the treatment of PPD with acupuncture.

\section{METHODS}

Participant or population: Patients with postpartum depression who have clear diagnostic criteria.

Intervention: The treatment group will be dominated by acupuncture(including electroacupuncture, warm acupuncture, ear acupuncture, etc.), acupuncture combined with drug therapy or psychotherapy, acupoint selection, acupuncture manipulation, needle retention time and course of treatment are not limited.

Comparator: The control group will be a single use of medium and Western medicine, psychotherapy or comfort therapy.

Study designs to be included: Randomized Controlled Trials (RCTs).

Eligibility criteria: Randomized controlled trials of acupuncture treatment of postpartum depression published from the inception of databases to May 2021 will be searched.

Information sources: From databases of CNKI, WANGFANG, SinoMed, VIP, PubMed, Web of Science, MEDLINE, EBSCOhost, the Cochrane Library and OVID.

Main outcome(s): The primary outcome indicator will be the effective rate(ER), and the secondary outcome indicators will be the Hamilton depression scale (HAMD) score, the Edinburgh postnatal depression scale(EPDS)score, and TCM syndromes Score, serum estradiol(E2)level, and occurrence of adverse reactions.

Quality assessment / Risk of bias analysis: The risk of bias of the included studies will be evaluated based on the built-in risk assessment tool of Review Manager5.3 software provided by the Cochrane Collaboration. The evaluation content includes selection, implementation, measurement, follow-up, report and others. The judgment results are given item according to the performance of the 
included literature in the above. If there is a disagreement, two authors will discuss it or be decided by a third one.

Strategy of data synthesis: The Review Manager 5.3 software provided by the Cochrane Collaboration will be used to conduct a meta-analysis of the included studies. Binary variable data will be used to calculate relative risk(RR)to facilitate clinical interpretation. Continuous variable data will be to calculate standardized mean difference(SMD) to eliminate the influence of different units or large differences in means. Both confidence interval(Cl) will be set to $95 \%$.

Subgroup analysis: Choose a fixed or random effect model based on the heterogeneity test results of the data. If $P>0.1$ and $I^{\wedge} 2<50 \%$, the fixed effect model will be selected. If $\mathrm{P} 50 \%$, the random effect model will be selected.If the heterogeneity is obvious, subgroup analysis, sensitivity analysis or descriptive analysis will be conducted.

Sensitivity analysis: The same as subgroup analysis. Choose a fixed or random effect model based on the heterogeneity test results of the data. If $P>0.1$ and $I^{\wedge} 2<50 \%$, the fixed effect model will be selected. If $\mathrm{P} 50 \%$, the random effect model will be selected.If the heterogeneity is obvious, subgroup analysis, sensitivity analysis or descriptive analysis will be conducted.

Country(ies) involved: China.

Other relevant information: The Affiliated Hospital of Xuzhou Medical University.

Keywords: Acupuncture and Moxibustion; Postpartum Depression; Meta Analysis.

Contributions of each author:

Author 1 - Yan Pan.

Author 2 - Jin Xi.

Author 3 - Jingyu Liu.

Author 4 - Jie Shen.

Author 5 - Jie Cheng.

Author 6 - Youbing Xia. 